\title{
Developing the Foundation for Syndromic Surveillance and Health Information Exchange for Yolo County, California
}

\author{
Osama Chaudhary, MPH ${ }^{1,2,3}$ \\ ${ }^{1}$ University of Illinois at Chicago, School of Public Health \\ ${ }^{2}$ Yolo County Health Department \\ ${ }^{3}$ Sacramento County Department of Health and Human Services
}

\section{Abstract}

This report delineates Yolo County Health Department's process to ascertain its optimal methods of participation in syndromic surveillance and health information exchange. As a health department serving a county of just 200,000 residents, Yolo County Health Department needed to operate within strict financial constraints. Meaningful Use legislation enabled it to pursue both syndromic surveillance and health information exchange participation whilst complying with its budgetary restrictions. The Health Information Technology for Economic and Clinical Health (HITECH), a segment of the American Recovery and Reinvestment Act of 2009, has incentivized the 'Meaningful Use' of electronic health records (EHRs) by providing incentive reimbursements and non-compliance penalties. The Meaningful Use of EHRs is to take place over 3 Stages: Stage 1 has begun, Stage 2 is imminent, and Stage 3 is currently being discussed.

Having been solicited by both health information exchange and syndromic surveillance options which were cost-prohibitive, Yolo County Health Department focused attention on BioSense 2.0, a Meaningul Use-ready and virtually free syndromic surveillance program developed by the Federal Centers for Disease Control and Prevention. In collaboration with Sacramento County Department of Health and Human Services, and with support from several other area counties, Yolo County Health Department submitted a Funding Opportunity application for BioSense 2.0 regional implementation. Through this collaboration, Yolo County Health Department has begun participating in the formative stages of the Sacramento Area Center for Advanced Biosurveillance (SAC-B). Via SAC-B, Yolo County Health Department will be able to participate in syndromic surveillance in the BioSense 2.0 program, and simultaneously expand its electronic health data sharing towards a more comprehensive health information exchange.

Lastly, over the course of these projects, three other methods of participating in electronic health data sharing became available to Yolo County Health Department: all three methods were the direct result of Meaningful Use legislation.

Key words: county health department; health information exchange; meaningful use, syndromic surveillance 


\section{Introduction}

Yolo County sits upon 1,021 square miles of agricultural terrain directly to the west of Sacramento, the State Capital of California. Most of Yolo County's 200,000 residents reside in one of four incorporated cities: Davis, Woodland, West Sacramento, and Winters. Some of the recognizable institutions in the county include the University of California, Davis, ranked in US News and World Report's Top 40 Universities, the Port of Sacramento, and the Triple-A baseball affiliate of the Oakland Athletics, the Sacramento Rivercats [1]. Yolo County is the ranked $7^{\text {th }}$ out of 58 California counties for Overall Health, boasting a $76 \%$ high school graduation rate, a 93\% rate on residents' access to healthy foods, a $16 \%$ rate of residents without health insurance, and an equally low $16 \%$ rate of physical inactivity in residents [2].

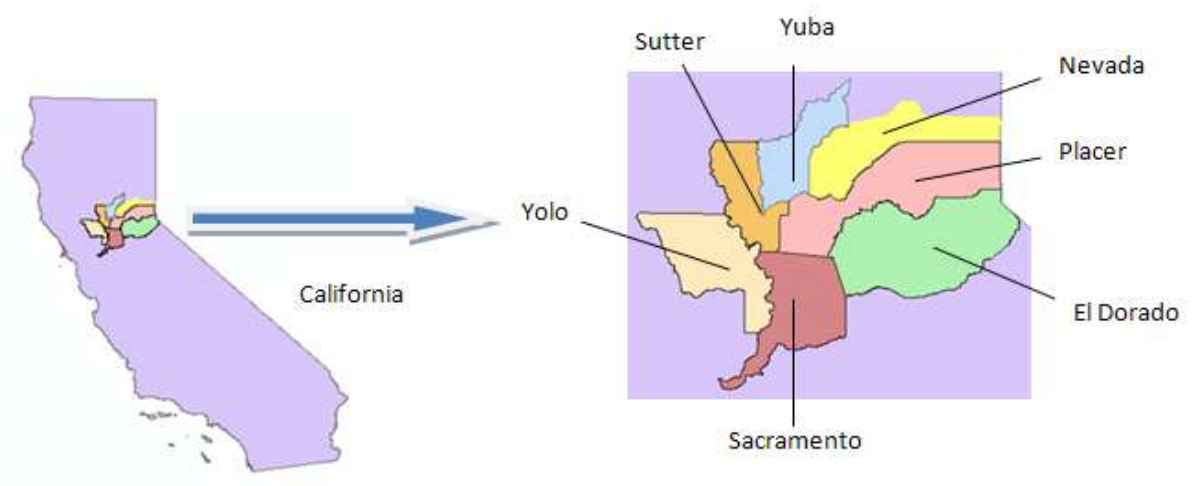

Figure 1: California and counties of the Greater Sacramento Area

Yolo County Health Department, located in Woodland, is composed of 9 divisions: Communicable Disease, Environmental Health, Indigent Health Medical Services, Public Health Education, Public Health Emergency Preparedness, Public Health Nursing, Public Health Laboratory, Vital Records, and WIC: Women, Infants, \& Children. In May of 2012, the two projects detailed in this report were initiated under the supervision of both the Division of Public Health Emergency Preparedness Supervisor and the Yolo County Health Department Epidemiologist.

\section{Purpose and Objectives}

The first project was to ascertain the optimal methods for Yolo County Health Department to participate in Health Information Exchange; the second was to ascertain the optimal methods for Yolo County Health Department to engage in syndromic surveillance.

Health Information Exchange (HIE) is the process of reliable and interoperable electronic health record (EHR) sharing, conducted in a manner that protects the confidentiality, privacy, and security of the information. The American Recovery and Reinvestment Act of 2009 (ARRA), commonly referred to as The Stimulus or The Recovery Act, allotted over $\$ 155$ billion for the purpose of enhancing healthcare delivery, with over $\$ 33.5$ billion provided for the pupose of optimally utilizing information technology in the healthcare delivery process. The crux of 
information technology's role in healthcare is the use of EHRs. An EHR is a "longitudinal electronic record of patient health information generated by one or more encounters in any care delivery setting. Included information are patient demographics, progress notes, problems, medications, vital signs, medical history, immunizations, laboratory data and radiology reports" [3]. ARRA has incentivized utilization of EHR transmission through the use of payment distribution and non-compliance penalties associated with deadlines for which "Meaningful Use" of EHRs must be fulfilled in healthcare organizations.

This is thus an extremely exciting, yet also possibly distressing, time for healthcare organizations. Concerns about meeting Meaningful Use requirements and deadlines may initially overshadow the vast benefits that Meaningful Use adoption of EHRs will provide for both providers and patients. An EHR's use can actually be as simple as a laboratory electronically transmitting test results to a clinic.

As defined by the Federal Centers for Disease Control and Prevention (CDC), syndromic surveillance is the use of "individual and population health indicators that are available before confirmed diagnoses or laboratory confirmation to identify outbreaks or health events and monitor the health status of a community" [4]. Syndromic surveillance conductors can now also leverage Meaningful Use regulations in seeking to obtain data from healthcare providers.

\section{Literature Review}

Yolo County Health Department required the planning of its potential HIE and syndromic surveillance participation to take into account it's extremely limited, and virtually absent, financial resources. The resultant quest for seeking potential sources of cost-free resources began with perusing such options as provided for through federal legislation.

ARRA's Health Information Technology for Economic and Clinical Health (HITECH) Act created incentive payments, to be distributed in the form of Medicaid and Medicare reimbursements, to Eligible Providers (EPs), Eligible Hospitals (EHs), and Critical Access Hospitals (CAHs), pending their demonstration of Meaningful Use of EHRs. Meaningful Use was designed to be demonstrated in 3 Stages.

Stage 1 reporting for Meaningful Use of EHRs began in 2011. Stage 2 reporting will begin in Fiscal Year 2014 for EHs and CAHs, and Calendar Year 2014 for EPs. Stage 1's most direct impact with regards to Public Health was its requirement that one of the following three capabilities was addressed by EPs, EHs, and CAHs: syndromic surveillance, Immunization Registries, or Electronic Laboratory Reporting [5]. Stage 2's most direct impact with regard to Public Health is that all three of the previous Public Health components have been proposed to be made mandatory. In addition, Stage 2 has been proposed to include reporting to a cancer registry as a mandatory fourth Public Health component. Finally, Stage 2 has been proposed to delineate mandatory technological requirements with which these four Public Health components should be fulfilled [5].

The Meaningful Use Objective for syndromic surveillance is the capability to submit electronic syndromic surveillance data to public health agencies except where prohibited, and in 
accordance with applicable law and practice. The statutory authority is found in section 170.314(f)(3), with regards to public health surveillance, and in section 170.314(f)(4), with regards to electronic data transmission to public health agencies [5]. Stage 2's proposed selection of the technological requirement for electronic data exchange is Health Level 7 version 2.5.1 [6].

Stage 3 requirements for Meaningful Use will likely include extensive utilization of Clinical Decision Support systems, recording advanced directives for elderly patients, expansion of demographic data, increased delineation of drug interaction effects, and further emphasis on sharing care summaries $[7,8]$.

The preview of Stage 2 requirements was welcome news to Yolo County Health Department, providing a viable framework upon which to proceed on both the HIE and syndromic surveillance projects. Yolo County Health Department would be able to leverage the Meaningful Use requirements in order to facilitate the data exchange necessary for HIE and syndromic surveillance, whilst simultaneously operating within their budgetary constrictions.

\section{Methods and Analysis}

At the time of the commencement of the projects, Yolo County Health Department had been asked to join the formative stages of what was tentatively being termed the North East Bay Health Information Exchange Services. Other areas being courted for involvement included Napa County, Solano County, and Sonoma County: all lay eastward from Yolo County.

Although the invitation for the North East Bay HIE Services group was Yolo County Health Department's first formal brush with potential involvement in HIE, and thus may have appeared enticing, two aspects of the invitation prompted a cautious approach. The first was that Yolo County Health Department was being asked to contribute $\$ 3,000$ in initial participation fees, with likelihood of recurring annual fees. The second factor was that most of Yolo County's residents and healthcare organizations were either based in, or operated towards, Sacramento, which is due west. After consultation with the Yolo County Health Department Director, the Yolo County Health Department Fiscal Advisor, and the Coordinator for the North East Bay HIE Services, it was decided that no imminent action would be taken, due both to the requested fiscal obligations and the lack of assurance that participation would benefit the residents of Yolo County.

Assessment was simultaneously being undertaken of syndromic surveillance possibilities. An electronic data vendor, Health Monitoring Systems, had been soliciting Yolo County Health Department for its business for several months. Fiscal constraints, however, once again proved prohibitive, and curtailed serious consideration regarding purchase of the syndromic surveillance programs bring proffered.

Fortuitously, however, BioSense 2.0, a CDC-led syndromic surveillance program, began to emerge as not only an extremely viable, but potentially extremely beneficent, option for Yolo County Health Department's future syndromic surveillance undertaking. 
BioSense 2.0 is a "[national public health surveillance system for the early detection and rapid assessment of potential public health threats. By using statistical aberration detection methods on health data supplied to health departments from a variety of sources, public health officials are able to identify and investigate anomalies both temporal and spatial, providing insight on the health and potential threats to the health of communities and country]" [9].

CDC oversees the BioSense 2.0 program in cooperation with the Association of State and Territorial Health Officials, the National Association of County and City Health Officials, and the Council of State and Territorial Epidemiologists. BioSense 2.0 offers Meaningful Use-ready, and HIPAA-compliant, syndromic surveillance capabilities. Its monumental benefits include both the fact that it has the support of, and contributions from, the aforementioned national organizations, and that it is virtually cost-free.

As BioSense 2.0 utilizes the cloud computing structure, there are no hardware or software costs or requirements for participants. All data is stored in what is referred to as the cloud environment. This cloud environment is the result of remote servers, owned by Amazon Web Servers, hosting the information, whilst users can effectively query, manipulate, and analyze this information via internet connected computers.

As it became evident that BioSense 2.0 could be utilized for syndromic surveillance by Yolo County Health Department, the next ascertainment to be made was to determine both how beneficial it could be, and in which manner it might be most advantageously utilized. Just as preparation to assess these possibilities began, CDC announced a BioSense 2.0 Funding Opportunity.

Funding Opportunity Number CDC-RFA-OE12-1202, entitled "BioSense 2.0: Building State, Local, Tribal, and Territorial Surveillance Capacity to Enhance Regional and National AllHazards Public Health Situation Awareness" was announced at the end of May. The application deadline for the $\$ 900,000 / 3$-year grant, however, was June 26 , thus leaving essentially a three week turnaround time for funding opportunity investigation and application planning, design, creation, revision, and submission (with the additional bureaucratic requirements such as obtaining the associated departmental approvals).

As Yolo County is home to only 200,000 residents, it seemed unlikely that the BioSense 2.0 Funding Opportunity would be awarded solely to Yolo County Health Department for countylevel use. As part of the Sacramento Metropolitan Statistical Area, however, Yolo County is part of a 5,000 square mile area which is home to over 2.1 million residents. The decision was made to solicit collaboration from the surrounding areas, beginning with the most prominent in terms of population and public health significance, Sacramento County.

Upon consulting with representatives from Sacramento County Department of Health and Human Services, El Dorado County Health Department, Nevada County Health Department, Placer County Health Department, Sutter County Health Department, and Yuba County Health Department, it was agreed that a greater Sacramento area collaborative effort at both participating in BioSense 2.0 and applying for the BioSense 2.0 Funding Opportunity would be preferable to attempting these efforts at individual county levels. 
Epidemiologists from these and adjacent counties, representing over 2.5 million residents across 10 counties, had been meeting regularly in a regional, interactive, and supportive process; the foundation for the collaboration was thus already laid. The regional approach to syndromic surveillance, however, still required a vision for the future. As syndromic surveillance is but one aspect of biosurveillance, and the county health representatives did not wish to be limited in subsequent endeavors, it was decided that all facets of biosurveillance should be sought to be supported.

A new organization would be created: the Sacramento Area Center for Advanced Biosurveillance (SAC-B). Sacramento County Department of Health and Human Services (Sac County DHHS), being the most prominent organization amongst those participating, would be the lead agency in both forming SAC-B and in applying for the BioSense 2.0 Funding Opportunity.

A grant-writing team commenced intense communication with the BioSense 2.0 Redesign Support Team, seeking guidance for planning and writing the Funding Opportunity application. After soliciting Letters of Support from health officers of the pertinent counties (some were unable to submit the letters in time for the submission), the Funding Opportunity application was submitted on June 22.

\section{Results and Discussion}

The two projects, the HIE and syndromic surveillance assessments, thus partially merged into one project. As syndromic surveillance is a foundation of biosurveillance, and as biosurveillance is a significant segment of HIE, this was a favorable, and possibly to-be-expected, outcome. In order to assess Yolo County Health Department's future in HIE, an HIE survey was created and sent to over 300 of Yolo County Health Department's partner organizations in healthcare and/or community service. Responses to this survey were still being received and collated as of the end of July, 2012.

Within two and a half months, however, Yolo County Health Department has progressed from regarding syndromic surveillance as simply a goal for which research should be undertaken, to membership in a regional collaboration for biosurveillance. As of the end of July, 2012, the Funding Opportunity application had still not been responded to by the CDC. Members of both Yolo County Health Department and Sac County DHHS were optimistic, however.

Despite the absence of notification regarding the Funding Opportunity application's status, SAC$\mathrm{B}$ began coalescing. Health officers and epidemiologists from the participating counties had been joined by representatives from Butte, Shasta, and San Joaquin Counties in discussing how the regional approach to BioSense 2.0 might be undertaken. To this end, relevant guidelines were collected from Tarrant County, Texas, a national leader in the regional approach to biosurveillance. Sample Data Use Agreements, material regarding HIPAA concerns from potential data suppliers, and a delineation of the various options for monitoring BioSense 2.0 for anomalies, were distributed to the various counties. 
SAC-B will leverage existing inter-stakeholder goodwill, Meaningful Use requirements, fiscal and quality-of-care improvements, standards and interoperability, data aggregation services, and member expertise to recruit and guide local electronic data providers for BioSense 2.0 participation. The objective of the SAC-B is to develop into a center of excellence which implements best practices that facilitate early detection and response to novel infectious disease pathogens, emerging diseases, disease outbreaks, bioterrorism, and urgent care departments.

Thus SAC-B will serve as the foundation for developing a thriving Health Information Exchange for the Greater Sacramento Area. This process is the type of regional multi-stakeholder effort that the Office of the National Coordinator for Health Information Technology has envisaged in its plan to form a Nationwide Health Information Network [8].

Finally, during the undertaking of these projects, the California Reportable Disease Information Exchange (CalREDIE), the California Immunization Registry (CAIR), and the California Cancer Registry (CCR) all became Meaningful Use-ready. These organizations were thus able to provide electronic health data regarding electronic laboratory reporting, immunization registry, and a cancer registry, respectively. Thus, Yolo County Health Department now has avenues for participating in electronic health data exchange for all four of the proposed Stage 2 Meaningful Use Public Health components.

\section{Public Health Significance}

Syndromic Surveillance is gaining acceptance as a crucial, if not necessary, tool, in early outbreak detection and bioterrorism prevention. Characteristics of the greater Sacramento area lend support to the need for a regionalized syndromic surveillance approach.

"The greater Sacramento area has experienced overall population growth throughout the recent economic recession. This includes a large population of seasonal migrant farm workers who reside in migrant camps throughout the region. Health inequity in access to care and health outcomes may increase incidence and propagation of novel or emerging disease transmission in such sub-populations.

The area is home to the Sacramento International Airport, the international Port of Sacramento, four interstate freeways (I-5, I-505, I-80, I-50) and numerous other highways and railways. Routine travel to and from Nevada, Oregon, the San Francisco Bay Area, Los Angeles, and Mexico, involving thousands of travelers, hazardous materials, and freight occurs daily.

The area is also home to the University of California at Davis, California State University at Sacramento and a number of other colleges and universities. These institutions are at increased risk of introduction of novel or emerging disease threats through international travel to and from campuses or may themselves present as targets for bioterrorism.

The swath of agricultural land surrounding the City of Sacramento produces a significant volume of the food produced in the US. Rice, tomatoes, fruit, and a variety of nut, vegetable, and grain crops originate in the area. As a result, large and potentially vulnerable food processing, storage, and distribution centers are located throughout the region and may present an intentional or 
unintentional opportunity for large-scale food contamination and food-borne disease transmission.

Finally, the State Legislature and 22 State governmental agencies are located in Sacramento. Federal agencies are also located in and around the region. The largest US Postal Service postal center in Northern California (one of three such centers in the entire State) is also located in the Sacramento area'"[10].

\section{Limitations}

The chief limiting factor of these two projects was dearth of time for completion. Health Information Exchange is a complex endeavor, requiring cooperation and coordination amongst a multitude of organizations on a multitude of levels, including two significant obstacles: legal and fiscal determinations. Thus, Yolo County Health Department will continue to communicate with its partner healthcare organizations in an attempt to hone a path towards increased HIE participation and/or recruiting. The optimal route is through participation in SAC-B and the leveraging of Meaningful Use legislation.

SAC-B had been serendipitously birthed partly out of the major time limitation of the syndromic surveillance project, as it was evident that collaboration amongst the area counties was necessary in order to increase chances for receiving the Funding Opportunity. SAC-B will now, however, continue to proceed towards active and collaborative regional biosurveillance activities in spite of the result of the Funding Opportunity application, and Yolo County Health Department thus has an avenue through which to develop syndromic surveillance capability and HIE participation.

\section{Acknowledgements}

The author would like to thank Dr. Cassius Lockett, Tim Wilson, MS, and Dana Carey, BS, for their support and guidance in this project. Dr. Lockett and Mr. Wilson co-designed and coauthored the CDC Funding Opportunity referenced in this report; Ms. Carey supervised each phase of the project.

The author would also like to thank Dr. Edward Mensah and Dr. Kshitij Nawal for their oversight and support during this project.

\section{Conflicts of Interest}

The author declares that he has no conflicts of interest.

\section{Correspondence}

Osama Chaudhary, MPH

12952 Riley Ct.

Rancho Cucamonga, CA 91739

Email: osamachaudhary@gmail.com 
Developing the Foundation for Syndromic Surveillance and Health Information Exchange for Yolo County, California

\section{References}

1. U.S. News \& World Report. National University Rankings. Available at: http://colleges.usnews.rankingsandreviews.com/best-colleges/rankings/nationaluniversities/data/page+2. Accessed June, 2012.

2. Robert Wood Johnson Foundation. County Health Rankings \& Roadmaps. Available at: http://www.countyhealthrankings.org/app/california/2012/yolo/county/1/. Accessed July, 2012.

3. Healthcare Information and Management Systems Society. EHR: Electronic Health Record. Available at: http://www.himss.org/ASP/topics_ehr.asp. Accessed July, 2012.

4. Centers for Disease Control and Prevention. Syndromic Surveillance. Available at: http://www.cdc.gov/ehrmeaningfuluse/Syndromic.html. Accessed July, 2012.

5. Department of Health and Human Services. Centers for Medicare \& Medicaid Services. Medicare and Medicaid Programs: Electronic Health Record Incentive Program: Final Rule.

6. Department of Health and Human Services. Centers for Medicare \& Medicaid Services. Medicare and Medicaid Programs; Electronic Health Record Incentive Program-Stage 2; Proposed Rule.

7. Mosquera, M. First Glimpse at Meaningful Use Stage 3 Measures. Government Health IT. Available at: $\quad$ http://www.govhealthit.com/news/health-it-panel-explores-draft-mu-3measures. Accessed July, 2012

8. iHealthBeat. Policy Committee Talks About Proposals for Meaningful Use Stage 3. Available at: http://www.ihealthbeat.org/articles/2012/8/3/policy-committee-talks-aboutproposals-for-meaningful-use-stage-3.aspx. Accessed August, 2012.

9. Kass-Hout, T., and Xu, Z. BioSense 2.0 Analytics: Change Point Analysis. June, 22, 2012. Available at: https://sites.google.com/site/biosenseredesign/community-forum. Accessed July, 2012.

10. Chaudhary, O., Lockett, C., and Wilson, T. Sacramento County Department of Health and Human Services, Public Health Division, Epidemiology and Disease Control. BioSense 2.0: Building State, Local, Tribal, and Territorial Surveillance Capacity to Enhance Regional and National All-Hazards Public Health Situational Awareness. Funding Opportunity Number CDC-RFA-OE12-1202. June 26, 2012. 